\title{
Scarcity Perception Personality Theory
}

\section{Dean Stalnaker $\mathbf{R}^{*}$}

South Iredell High School, Troutman, NC, USA

\begin{abstract}
A personality theory focuses on variations among individuals. In forming a personality theory, it is essential to construct an image of the individual and the psychological processes that comprise that picture. A personality theory aims to investigate the psychological variations that form our personality. The primary focus of personality theory is comprised on the concept of general scarcity. Scarcity is the idea of having a shortage of an item. Scarcity is a wanting or lack of something that we have determined that we must have. Scarcity is a term that is often used in the field of economics, which drives the forces within various types of economic systems. However, our perception of scarcity dominates more than just our financial system it has a much larger role in our lives. Within theory of personality, there is a biopsychosocial aspect of our existence associated with our perception of scarcity that forms our personality and determines our view of oneness with our multi-environments. How we perform in our environments determines the shape, direction, and stability of those conditions. There is an order of unity between our perception of scarcity and environments.
\end{abstract}

Keywords: Scarcity; Perception; Personality; Bio psychosocial; Muilt-environments

\section{Introduction}

In our world today we are faced with many conflicts: biological, ecological, social, psychological, economic, and political. These internal and external conflicts could all be a side effect of our perception of scarcity $[1,2]$. The interactions of human kind within our environments are related to how we perceive the concept of scarcity. An individual's view of their particular environment depends on their perception of scarcity. The person chooses how they will see the world and act based upon how they formulate personal constructs either faithful to their intrinsic cognitive passions or not. How the individual views the world will shape their overall perception of that world or environment and affect their behavior within that environment. Theory suggests that their perception of scarcity is the catalyst that drives their personality and thus their behaviors. The fundamental question is when does this process start?

\section{Formation of Factors in Personality}

Early in an individual's life, they begin the shaping of motivational constructs, which have been established as an essential part of the formation of personality. The concepts of personality traits and motivational constructs have not always been detailed. Nevertheless, they are vital for defining the person. Personality describes what the person is like and the construct gives us an indication of what the person wants to become [3]. This conceptual relationship between personality characteristics and motivational constructs are connected to an interplay between nature and nurture.

Bleidorn et al. identified that modern personality theories show variations in their expectations and dissection of the correlation between personality theories and motivational constructs [3]. The researchers examined the genetic and environmental sources of the interaction of the Five Factor Model and major life goals contemporarily and over time in an attempt to produce a more meaningful assessment of the different assumptions expressed in the FFM as conflicting to socio-analytic understandings. Bleidorn et al. research consisted of evaluating traits and goals being evaluated twice over a five-year period with a sample group of 217 identical and 112 fraternal twin pairs in association with Bielefeld Longitudinal Study of Adult Twins [3]. Bleidorn et al. found that only $30 \%$ of variations for life goals were genetic [3]. The $30 \%$ variation suggests that $70 \%$ of the variation of the groups were due to a non-shared perception of environmental factors. The researchers revealed reciprocal genetic and environmental factors on personality traits. As we evaluated the variations of personality traits and life goals, we cannot ignore the influence of both nature and nurture on these factors. However, the variations being controlled by external environmental forces are far more impressionable. Believes what the researchers did not examine was the participants' perception of scarcity on their view of motivation constructs and the adaptation of their personality traits. Individuals are motivated to behave in certain ways based on their interpretation of scarcity within their environments. The feeling of both inferiority and superiority can be contributed to the concept of scarcity. An individual strives to achieve their full potential is related to the cognitive impact of scarcity. Consider how the scarcity of time influences our actions. How we respond to time is based on our perception of the scarcity of that time. A person struggles with ideal self, and real self could be associated with their perceived notion of their abilities to progress as they understand the impact of scarcity. The perception of scarcity has the power to limit a person's ideology of motivation. Consider scarcity of physiological abilities how a person perceives physical limitation or scarcity of these abilities can impact their personality and be manifested in their behaviors. The cognitive processes of managing scarcity maybe the difference between a mass destructive personality or a mass creative personality.

Kristofferson, McFerran, Morales, and Dahl examined the adverse impact of scarcity as it relates to exposure to limited-resources and how the perception of scarcity can cause aggression [4]. This study is based on an individual's perception of scarcity and how it can impact aspects of our society. Within our formation of personality from the nurture side how we interpret the impact of scarcity in various environments will influence our overall behaviors. Kristofferson et al.

*Corresponding author: Dean Stalnaker, South Iredell High School, Troutman, NC, USA, Tel: +1 704-528-4536; E-mail: rstalnaker@iss.k12.nc.us

Received June 06, 2018; Accepted June 29, 2018; Published July 06, 2018

Citation: Stalnaker RD (2018) Scarcity Perception Personality Theory. Arts Social Sci J 9: 387. doi: 10.4172/2151-6200.1000387

Copyright: (๑) 2018 Stalnaker RD. This is an open-access article distributed under the terms of the Creative Commons Attribution License, which permits unrestricted use, distribution, and reproduction in any medium, provided the original author and source are credited. 
demonstrated how companies would use scarcity incentives when a product or event is limited in obtainability [4]. When an individual is placed in an environment that presents circumstances under which the mere exposure to the conditions can set in motion actual aggression that displays even outside the sphere of influence of the good being in the mainstream. The researchers suggested that the procedure that fundamentally affects the exposure to limited amount preferment advertising to interpret the interaction with other people as a competitive threat to acquire the desired product and this causes the people to be aggressive.

Kristofferson et al. conducted seven studies using various behavioral measures of aggression to explain this harmful response to scarcity [4]. Is this perception of scarcity only limited to consumer items? The perception of scarcity shapes the individual's personality in various situations and interaction with the formation of individual constructs on how we will demonstrate behavior in our societal environments. The individual perception of scarcity is not limited to economic goods, but also to our interactions with others on multiple levels. As our perception of scarcity influences our cognitive processes, social interactions, and viewpoint of our environments. It can cause the individual to evaluate the value of each interaction and the importance of the frequency of those interactions. We do not just simply respond to scarcity, but scarcity as an external environmental force shapes our personality. This process begins early in our childhood and continues to shape us throughout our adulthood. The perception of scarcity can change as we go through many developmental stages of life. The impact of our perception of scarcity causes changes in our personality.

Consider the impact of scarcity on childhood. A child can be taught about the concept of scarcity by observational learning from parents or by imitation of behaviors of adults they are surrounded by. The child's perception of scarcity can cause the child to demonstrate behaviors of selfishness or generosity based on their understanding. The behaviors of selfishness and generosity become part of their personality shaping the construct they form. The constructs will be used in many different environments to circumscribe their interpretation of appropriate behavior to apply. Roux, Goldsmith, and Bonezzi suggested that people are often reminded of scarcity throughout their life [5]. There is little information about the psychological progressions that these reminders create. The researchers suggested that these reminders of scarcity instantiate competition, which causes the individual to make decisions toward an advantage that would protect their self-interest. This concept of self-interest depends on the personal construct formed based on their perception of scarcity. The construct could be one of selfish behavior or one of generosity. The response depends on what is best for their welfare. The more the individual is exposed to various situations within their environments where scarcity plays a dominant role the more their personality traits such as selfishness and generosity will be reinforced. Keep in mind that if the individual's perception of scarcity changes then their behavior can be affected. Roux et al. suggested that the data produced in their study could be a first step in understanding why scarcity may support variations in behavioral responses in different environmental settings throughout one's lifespan [5].

Raymond Cattell presented sixteen personality factors that were common in each. It could be possible that each factor is influenced by the individual's perception of scarcity. Scarcity can impact the level of our apprehension. Feelings of insecurity versus security could be determined by how we perceive the scarcity of money, companionship, or sociability. Consider dominance concerning an individual's perception of scarcity. If the individual observes scarcity in a way, there will never be enough they are more likely to be overly forceful due to high levels of selfishness. If the individual views scarcity as a motivator to share or conserve they may exhibit higher levels of generosity in various situations in society. Cattell's psychological evaluation questionnaire measures atypical and pathological traits to produce a multidimensional characterization of an individual based on sixteen factors [6]. The individual perception of scarcity is the motivator by which the individual responses to the questions surrounding the sixteen personality factors

\section{Scarcity's Impact on Minorities, Culture, Socioeconomic Status, Gender, and Sexual Orientation}

Scarcity is more likely to be observed in societies where there is a greater demand for an object that there is a supply for that object. When this event occurs, something happens to us on a biopsychosocial level. Individuals desire to hang on to their youth, but this is not possible because of a scarcity of time and the aging process. Often we find ourselves in relationships where there is some scarcity of affection or intimacy. Another example of scarcity and its effect on society is in the area of life experiences, contentment, and inner peace. Consider how much mental and physical energy is spent when you have limited means to support your family. Think about how certain minority groups have to manage their lives based on discrimination, prejudice thoughts, and stereotypes because there is a scarcity of understanding and compassion in our society [7].

Nelson and Morrison researched the symptoms of resource scarcity which involves decision making based on how food and finances impact our preferences for probable companions [8]. The researchers identified evidence of male inclinations for female body weight pursue a consistent cross-cultural prototype which includes cultures with limited reserves, women that appear heavier are favored. However, in cultures with abundant resources, women who appear lighter in weight are preferred. The researchers provide a social cognitive description for these results based on an individual's perception of scarcity. Nelson and Morrison conducted four studies consisting of 1,176 participants [8]. The researchers examined the prospect that this cross-cultural phenomenon begins at the individual level with their perception and experiences with scarcity. The evidence discovered by the researchers suggested that men that felt a greater degree of scarcity with various resources such as food prefer heavier females than males that had levels of lower scarcity concerns to different resources. Nelson and Morrison identified these cross-cultural patterns as part of a psychological process that begins on an individual level based on a perception of scarcity [8]

Within the field of cultural psychology, there are two fundamental factors at work: (1) people are different in various cultures and (2) some of their characteristics are universal across cultures. The findings of Nelson and Morrison research confirms the validity of both of these fundamental factors. The researchers began their study motivated by cross-cultural variances but identified a psychological mechanism that operates across-cultures. The perception of scarcity is universal across cultures and is a mental process that determines how we demonstrate behaviors in a given situation. The concept of scarcity is not limited to just finances and food but could be present in all aspects of our lives. Consider Maslow's Hierarchy of Needs.

An individual's perception of scarcity is the primary motivator on how they achieve each level of the prescribed needs: physiological, safety, belonging, esteem, and self-actualization. Datta suggested that Maslow's Hierarchy of Needs centered around the individual [9]. Maslow's theory deals with how we interact with our environment 
and that self-actualization concentrates on the individual's progress of finding their full potential. However, each step we take is motivated by a perception of scarcity, which is internalized and experienced through the process of achieving self-actualization. The perception of scarcity is ever-present as individuals reach the levels of needs as best they can across-cultures.

Yearby presented a study that concentrated on racial inequities in access to health care [10]. In the research Yearby identified a 12-yearold African American boy that died due to a toothache because he did not have $\$ 80$ to have a routine tooth extraction [10]. His Medicaid Insurance would have paid the remainder of the tooth extraction. The young African American boy ended up in the emergency room and endured two brain surgeries, and stayed in the hospital for six weeks, which cost $\$ 250,000$. However, in the end, the young boy died due to a brain infection caused by the spread of bacteria from the abscess in his mouth. The young boy did not die due to the treatment he received based on his race. He died because of the scarcity impact of income disparity on minorities [10]. Yearby identified ethnic minorities and socioeconomic classes that are faced with dealing with a health care system that is based on ability to pay [10]. The system establishes a preference of wealth over need. This method of distribution is the display of structural bias, which puts greater value on wealth over what should be common. A perception of scarcity motivates this system. Ethnic minorities in society tend to suffer the most which is usually determined by the oligarchy's perception of scarcity.

Cronk discussed the concept of gender preferences from an evolutionary viewpoint [11]. In his article, Cronk focused on the Trivers-Willard Hypothesis, which estimated that the idea of natural selection had preferred parents that prejudice investment to the sex with the greatest reproductive prospects. Due to a perception of resource richness of scarcity often have more significant impact on male than the female reproductive accomplishment [11]. The TriversWillard Model suggests that natural selection will most often prefer parents that pick males when scarcity conditions are good and females when scarcity conditions are poor. Accordingly, due to the evolutionary force of natural selection, more males are born when resources are more plentiful, and females are born when resources are more scares. However, empirical data of this hypothesis are inconclusive to the terms of the suitableness of Trivers-Willard's methods and their application to the model [11].

The Trivers-Willard Model suggests that evolution or natural selection could play a role in the perception of scarcity of female mammals can adapt offspring sex relative amount in response to their parental needs [11]. It is possible for the idea of scarcity to be part of our evolutionary nature that is innate within each human. However, it is the nurture of environmental forces that shape our perception of scarcity. As our perception of scarcity forms so does our personality.

\section{Scarcity Perception Personality Theory Compared to Other Personality Theories}

Comparing the Scarcity Perception Personality Theory to other methods encompasses both sides of nature and nurture. Consider Freud's Theory of Instinctual Drives, which is developed with an interaction of instinct and environmental forces. Freud introduced drives such as food, sex, and aggression. The principle of these instincts are motivated by some understanding of scarcity and as that perspective of scarcity evolves so does the importance of these instinctual drives. Parents become an important nurturing tool as the child moves through the steps of the psychosexual stages, which is crucial to normal and abnormal development. Scarcity, due to its evolutionary nature could be part of Freud's unconscious processes [12].

From a nurturing base, consider the Social Cognitive Learning Theory. Bandura suggested that humans learn by observations, imitation, and modeling. The Learning Theory proclaims that humans will make many observations of other people's actions and these observations will have a cognitive impact on the observer. The individual will weigh the results of the behaviors they have observed and determined if they can imitate these actions and if these measures can benefit them in any way. A cognitive benefit analysis based on loss or gain influenced by their perception of scarcity. The action the individual chooses to take could be one of selfishness, generosity, or aggression to name a few. Consider the concept of self-efficacy, which is an individual's belief they can succeed in a given situation. The motivation to try to achieve must first be motivated by the goals the person has established. Their perception of scarcity could be the key to that motivation.

These few personality theories have presented are based on biopsychosocial factors some being more based on internal factors than external factors that drive our development of personality. We suggest the approaches that tend to be more one sided than the others are lacking an understanding of both the internal and external forces that are working together to establish who we are. Believes the Scarcity Perspective Personality Theory embraces both forces suggests that a basic understanding of scarcity is innate. Just as a child is born with the rooting reflex we are born with an idea of scarcity. Maybe the baby roots because they have an idea of scarcity of life and the importance of feeding.

Freud discussed the interaction of the Ego and Superego using terms such as pleasure principle, reality principle, and defense mechanisms. However, what is the motivator behind these drives? Could it be an internal conflict between consciousness and unconsciousness with a common motivator of scarcity? The Neo-Freudians discussed internal conflicts, archetypes, and inferiority. Could scarcity be the internal drive that contributes to these levels of feelings associated with inadequacies and perception of cultural factors?

The humanists used terms such as full potential, self-concept, and self-actualization. Could the pathway to finding full potential, achieving higher levels of the hierarchy of needs, and discovery of harmony between real-self and ideal-self be based on one's perception of scarcity? Think what is lacking in many of these theories of personality is an understanding of oneness that exists between nature and nurture. Nature and nurture can have both positive and negative effects based on the individual's perception of scarcity. That a balanced individual has a positive interpretation of what scarcity is and the forces of nature and nurture are also balanced in their life. When there is a negative interpretation of scarcity, there is an imbalance between nature and nurture in the person's life. Think with these other personality theories a primary motivator is missing, and that is the perception of scarcity.

\section{Conclusion and Future Prospects}

\section{Basis idea of scarcity perception personality theory}

The basis theory is designed by observations and experience. As a former Marine and a police officer and currently a teacher, have seen individuals behaving at their best and worst, have seen people that have no mutual interest in other people stop and help others in need and have seen people hurt individuals that they have said they loved to take just a few dollars. Observed people who have plenty cheat and steal 
to obtain more. Worked cases where individuals have taken their own lives for reasons that seemed so small. It reflects on childhood growing up in a middle-class family with its various struggles. Watching mother and father work hard all their lives living pay check to pay check. At times we had to make sacrifices and do without certain items. Think with all the observations and experiences that are part of life have learned an appreciation for life, time, and emotions. Think with some of the individuals have observed they feared scarcity of not having enough time or enough "things" to go around. Think their perception of scarcity and how it influenced their personality was evident in their behaviors have also seen the better side of people humanity who are dedicating their lives to help others in many ways. Have seen how their behavior of goodness has shaped their perception of scarcity and their personality. The individual's perception of scarcity is vital for the shaping of the personality and finding their place in society. It is important as individuals that we learn that giving to others in various ways can change our perception of scarcity and lead to living a more abundant life.

\section{References}

1. Aşkun D, Çetin F (2016) How Do We Demonstrate Oneness as a Behavior? Operationalizing Oneness Through Scale Measurement. Journal of Spirituality in Mental Health 19(1): 34-60.

2. Bandura $A$ (2009) Social cognitive theory goes global. American Psychological Association 22: 504-506.
3. Bleidorn W, Kandler C, Hülsheger U, Riemann R, Angleitner A, et al. (2010) Nature and nurture of the interplay between personality traits and major life goals. Journal of Personality and Social Psychology 99: 366-379.

4. Kristofferson K, McFerran B, Morales A, Dahl D (2016) The Dark Side of Scarcity Promotions: How Exposure to Limited-Quantity Promotions Can Induce Aggression. Journal of Consumer Research 43: 56.

5. Roux C, Goldsmith K, Bonezzi A (2015) On the Psychology of Scarcity: When Reminders of Resource Scarcity Promote Selfish (and Generous) Behavior. Journal of Consumer Research 42: 615-631.

6. Cattell R, Cattell A, Cattell H, Russell M, Bedwell S, et al. (2002) Psych Eval Personality Questionnaire.

7. Novotney A (2014) The psychology of scarcity.

8. Nelson L, Morrison E (2005) The Symptoms of Resource Scarcity Psychological Science 16: 167-173.

9. Datta Y (2010) Maslow's Hierarchy of Basic Needs: An Ecological View Oxford Journal 9: 39-57.

10. Yearby R (2011) Racial Inequities in Mortality and Access to Health Care. Journal of Legal Medicine 32: 77.

11. Cronk $L$ (2007) Article: Boy or girl: gender preferences from a Darwinian point of view Reproductive Biomedicine Online 15(2): 23-32.

12. McLeod S A (2014) Theories of Personality. 